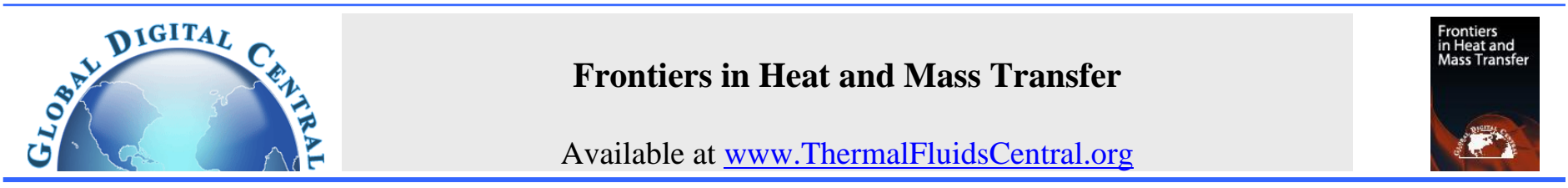

\title{
THE EFFECTS OF THERMAL RADIATION AND NON-UNIFORM HEAT SOURCE/SINK ON STRETCHING SHEET EMBEDDED IN NON-DARCIAN POROUS MEDIUM
}

\author{
Wubshet Ibrahim ${ }^{\mathrm{a}, *}$ and Bandari Shankar ${ }^{\mathrm{b}}$ \\ ${ }^{a}$ Department of Mathematics, Ambo University, Ambo, P.O.Box 19, Ambo, Ethiopia \\ ${ }^{\mathrm{b}}$ Department of Mathematics, Osmania University, Hyderabad 7, Telengana, India
}

\begin{abstract}
The Numerical analysis of magneto-hydrodynamics (MHD) boundary layer flow and heat transfer of incompressible, viscous and electrically conducting fluid is presented. The flow is due to continuously stretching permeable surface embedded in non-Darcian porous medium in the presence of transverse magnetic field, thermal radiation and non-uniform heat source/sink. The flow equations in the porous medium are governed by ForchheimerBrinkman extended Darcy model. A similarity transformation is used to transform partial differential equations into a coupled higher order non-linear ordinary differential equations. These equations are solved numerically using implicit finite difference scheme called Keller-Box method. The effects of the governing parameters on velocity and temperature are computed, analyzed and discussed. Moreover, the numerical results for the local skin friction coefficient and local Nusselt number are computed for various physical parameters governing the flow problem. It is found that increasing Darcy number accelerates the flow but increasing Forchhiemer number causes deceleration in the flow. The findings of the present study reveal that an increase in the radiation, heat source and Forchheimer number increases the thermal boundary layer thickness. The results under the limiting case were compared with the previously published work and found to be in good agreement.

Keywords: Heat Transfer, Boundary-Layer flow, Non-Uniform Heat source or Sink, Stretching Surface, Implicit finite difference (Keller-Box ) Method, Non-Darcy Porous Medium.
\end{abstract}

\section{INTRODUCTION}

The analysis of radiation effect has important applications in physics and engineering especially in space technology and high temperature processes. Radiation has a big impact on the boundary layer flow. However, very little is known about the effects of radiation on the boundary layer flow. Thermal radiation effect on the boundary layer may play important role in controlling heat transfer in polymer processing industry where the quality of the final product depends on the heat controlling factors to some degree. Radiative effect often observed in many engineering areas such as in electrical power generation, astrophysical flows, solar power technology, space vehicle re-entry, nuclear engineering applications and other industrial areas. Accordingly, researchers (Siddheshwar and Mahabaleswar (2005), Mahmoud (2009), Shateyi and Motsa (2010), Abel and Mahesha (2008), Prasad et al. (2010)) have examined the effects of thermal radiation on heat transfer over stretching sheet by considering different properties of a fluid. Moreover, when radiative heat transfer takes place, the fluid involved can be electrically conducting since it is ionized due to the high operating temperature. Therefore, it is important to examine the effect of the magnetic field on the flow. Many researches have examined the effects of thermal radiation on fluid flow and heat transfer in the presence of magnetic field. Accordingly, Pal (2011) studied the effects of thermal radiation and non-uniform heat source/sink on heat transfer over permeable stretching surface. Moreover, Abel and Mahesha (2008) also has conducted numerical study of the effects of thermal radiation on a boundary-layer flow of a micro polar fluid with temperature dependent viscosity and thermal conductivity over a stretching sheet through porous media. Still further, Elbashbeshy and Aldawody (2010) studied heat transfer over stretching surface by considering variable heat flux in the presence of a uniform heat source/sink. Furthermore, Pop et al. (2011) studied the radiation effects using Rosseland model on the flow of an incompressible viscous fluid over a flat sheet near the stagnation point.

The analysis of the flow and heat transfer induced by continuous stretching heated surface placed in the porous medium has received the attention of many researchers now days due to its important applications in many field of engineering disciplines. For example, applications of the porous medium includes, thermal insulation of buildings, heat exchanger, solar energy collector, geophysical applications, solidification of alloys, nuclear waste disposal, drying processes etc. More applications on porous medium are available in the book by Nield (2006)

Further, Elbashbeshy et al. (2010) investigated the unsteady boundarylayer flow over a porous stretching surface embedded in a porous medium in the presence of uniform heat source or sink. Their result indicates that the unsteadiness parameter increases the skin friction coefficient and the

${ }^{\dagger}$ Corresponding author. Email: wubshetib@yahoo.com 
local Nusselt number for different values of Prandtl number Pr. Moreover, Elbashbeshy and Bazid (2004) investigated heat transfer effect over an unsteady porous stretching surface embedded in a porous medium with variable heat flux in the presence of heat source or sink. Furthermore, Ishak et al. (2009) numerically examined the effect of Heat transfer over unsteady stretching permeable surface with prescribed wall temperature.

In all the above studies, the investigators are confined to Darcy's law which state that the volume-average velocity is proportional to the pressure gradient. The Darcy model is valid in a densely packed porous medium made up of uniform spherical particles under condition of low velocity and small porosity and permeability Pal and Chatterjee (2010). However, for relatively high velocity flow situations, the Darcy's law is inadequate for representing the flow behavior correctly since it does not account for the resulting inertia effects of the porous medium. In such case, the pressure drop has a quadratic relationship with volumetric flow rate. Therefore, the inclusion of non-Darcian effect term necessitate for more realistic solution of the problem. The inertia effect is expected to be important at higher flow rate and it can be accounted for the addition of a velocity squared term in the momentum equation, which is known as the Forchheimer's extension of the Darcy's law. Several investigators have considered the non-Darcian model to study the heat transfer rate on surfaces embedded in a porous medium. Accordingly, Takhar et al. (1998) studied the radiation-convection dissipative non-Gary gas flow in a non-Darcy porous medium using Keller-Box implicit finite difference schemes. Recently, Pal (2010) studied magnetohydrodynamic non-Darcy mixed convection heat transfer from a vertical heated plate embedded in a porous medium with variable porosity. All the above researchers studied the non-Darcy porous medium when the physical properties of a fluid is a constant. However, Pal and Mondal (2010) studied the effects of variable viscosity on MHD non-Darcy mixed convection heat transfer over stretching sheet embedded in porous medium. Some of the important references dealing with the concept discussed in the paper are found Abolbashari et al. (2014); Rashidi and Erfani (2012); Rashidi et al. (2014); Ibrahim (2016); Rashidi et al. (2012)

In all the above studies, the simultaneous effect of non-uniform heat source/sink, thermal radiation, magnetic parameter and porosity were not studied. Hence, the present study is aimed at filling this gap. The results of this study is useful in many engineering applications such as MHD generator, nuclear rectors, geothermal energy extractions etc.

\section{MATHEMATICAL FORMULATION}

Consider steady two-dimensional laminar boundary layer flow of an incompressible viscous fluid over a continuously moving stretching permeable surface embedded in non-Darcy porous medium in a quiescent incompressible electrically conducting fluid. The $\mathrm{x}$-axis is taken along the stretching surface in the direction of the motion with the slot as the origin as shown in fig.1.

The flow is subjected to a transverse magnetic field of strength $\mathrm{B}(\mathrm{x})$ which is assumed to be applied in the positive y-direction normal to the surface, thermal radiation and non-uniform heat source /sink. The induced magnetic field is assumed to be small compared to the applied magnetic field and is neglected. Moreover, applied electric field, Hall effect and Joule heating are neglected.

We also assume that the sheet is stretched with the stretching velocity $u_{w}(x)$ along $\mathrm{X}$-axis and the surface temperature of the sheet is $T_{w}(x)$. The fluid properties are assumed to be isotropic and homogenous. Darcy resistance and quadratic drag terms are taken in to account by considering constant permeability of the porous medium. In order to get the effect of temperature difference between the surface and ambient fluid, we consider the non-uniform heat source/ sink in the flow. Under these assumptions along with the boundary-layer approximations, the continuity, momentum and energy equations are respectively, as used by Abel

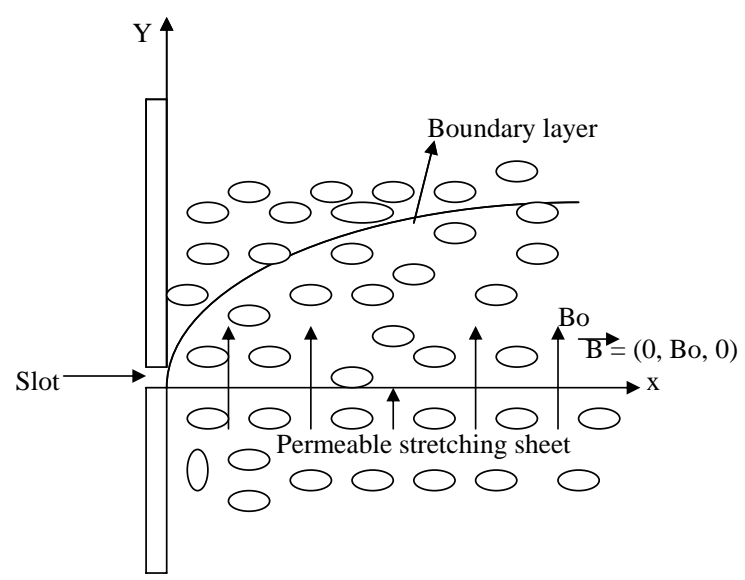

Fig. 1 Schematic Diagram for Physical Flow Model

and Mahesha (2008) given by

$$
\begin{aligned}
\frac{\partial u}{\partial x}+\frac{\partial v}{\partial y} & =0 \\
u \frac{\partial u}{\partial x}+v \frac{\partial u}{\partial y} & =v \frac{\partial^{2} u}{\partial y^{2}}-\sigma \frac{B^{2} u}{\rho}-v \frac{u}{k^{\prime}}-\frac{c_{b}}{\sqrt{k^{\prime}}} u^{2} \\
u \frac{\partial T}{\partial x}+v \frac{\partial T}{\partial y} & =\frac{k}{\rho c_{p}} \frac{\partial^{2} T}{\partial y^{2}}-\frac{1}{\rho c_{p}} \frac{\partial q_{r}}{\partial y}+\frac{q_{n}}{\rho c_{p}}
\end{aligned}
$$

with boundary conditions

$$
\begin{aligned}
u=u_{w}(x), \quad v & =v_{w}, \quad T(x)=T_{w}(x) \quad \text { at } \quad y=0 \\
u & \rightarrow 0, \quad T \rightarrow T_{\infty} \quad \text { as } \quad y \rightarrow \infty
\end{aligned}
$$

where $\mathrm{x}$ and $\mathrm{y}$ represent coordinate axes along the continuous surface in the direction of motion and normal to it, respectively; $u$ and $v$ are the velocity components along $\mathrm{x}$-axis and $\mathrm{y}$-axis, respectively; $v$ is kinematics viscosity; $\sigma$ is electric conductivity, $\mathrm{T}$ is the temperature inside the boundary layer, $c_{p}$ is the specific heat at constant pressure, $\rho$ is the density, $\mathrm{k}$ is the thermal conductivity, $k^{\prime}$ is the permeability of the porous medium; $c_{b}$ is the form of drag coefficient which is independent of viscosity and other physical properties of fluid but is dependent on the geometry of the medium; $T_{\infty}$ is the temperature far away from the stretching sheet with $T_{w}>T_{\infty}$, and $\mathrm{a}, \mathrm{b}$ are constants, $a>0, b \geq 0$.

The velocity of the sheet $u_{w}(x)$, the surface temperature of the sheet $T_{w}(x)$ and the transverse magnetic field strength $B$ are respectively defined as follows

$$
u_{w}(x)=a x, \quad T_{w}(x)=T_{\infty}+b x, \quad B=B_{0}
$$

The particular form of $u_{w}(x)$ and $T_{w}(x)$ represented in this paper has been chosen in order to devise a similarity transformation, which transform the partial differential equations (1)-(3) into a set of high order nonlinear ordinary differential equations. The equation of continuity is satisfied if we choose a stream function $\psi(x, y)$ such that

$$
u=\frac{\partial \psi}{\partial y}, \quad v=-\frac{\partial \psi}{\partial x}
$$

The mathematical analysis of the problem is simplified by introducing the following similarity transforms

$$
\eta=\sqrt{\frac{a}{v}} y, \quad \psi=\sqrt{a v} x f(\eta), \quad \theta(\eta)=\frac{T-T_{\infty}}{T_{w}-T_{\infty}}
$$

The term $v_{w}=-\left(\frac{v u_{w}}{x}\right)^{\frac{1}{2}} f(0)$ represents the normal velocity at the surface with $v_{w}>0$ for injection and $v_{w}<0$ for suction. 
The radiative heat flux in the $\mathrm{x}$-direction considered negligible as compared to y-direction. Hence, by using Rosseland approximation for radiation, the radiative heat flux $q_{r}$ is given by

$$
q_{r}=-\frac{4 \sigma^{*}}{3 k^{*}} \frac{\partial T^{4}}{\partial y}
$$

where $\sigma^{*}$ and $k^{*}$ are respectively the Stephan-Boltzmann constant and the mean absorption coefficient. We assume that the temperature difference with in the flow are sufficiently small such that the term $T^{4}$ may be expressed as a linear function of temperature. This is done by expanding $T^{4}$ in a Taylor series about a free stream temperature $T_{\infty}$ as follows:

$$
T^{4}=T_{\infty}^{4}+4 T_{\infty}^{3}\left(T-T_{\infty}\right)+6 T_{\infty}^{2}\left(T-T_{\infty}\right)^{2}+\cdots
$$

Neglecting higher-order terms in the above equation (9) beyond the first degree in $\left(T-T_{\infty}\right)$, we get

$$
T^{4} \cong 4 T_{\infty}^{3} T-3 T_{\infty}^{4}
$$

Thus substituting equation(10) in to equation(8), we get

$$
q_{r}=-\frac{16 T_{\infty}^{3} \sigma^{*}}{3 k^{*}} \frac{\partial T}{\partial y}
$$

The non-uniform heat source/sink, $q_{n}$, is defined as follows, as it is used in Pal (2011)

$q_{n}=\frac{k u_{w}(x)}{x v}\left[A 1\left(T_{w}-T_{\infty}\right) f^{\prime}+\left(T-T_{\infty}\right) B 1\right]$

where $A 1$ and $B 1$ are the coefficient of space and temperature-dependent internal heat source/sink, respectively. $A 1>0, B 1>0$ corresponds to internal heat generation and $A 1<0, B 1<0$ corresponds to internal heat absorption. Using the similarity transformation equation, the governing equations (1), (2) and (3)are transformed to the non-dimensional form as follow

$$
\begin{array}{r}
f^{\prime \prime \prime}+f f^{\prime \prime}-(1+F) f^{\prime 2}-\left(M+\frac{1}{D a}\right) f^{\prime}=0 \\
(1+R) \operatorname{Pr}^{-1} \theta^{\prime \prime}+f \theta^{\prime}-f^{\prime} \theta+\operatorname{Pr}^{-1}\left(A 1 f^{\prime}+B 1 \theta\right)=0
\end{array}
$$

With boundary conditions

$$
\begin{array}{r}
f(0)=s, \quad f^{\prime}(0)=1, \quad \theta(0)=1, \quad \eta=0, \\
f^{\prime}(\infty)=0, \quad \theta(\infty)=0, \quad \eta \rightarrow \infty
\end{array}
$$

Where $f(0)=s$ with $s<0$ and $s>0$ corresponds to injection and suction, respectively. $f^{\prime}$ and $\theta$ are the dimensionless velocity and temperature respectively, $\eta$ is the similarity variables, the prime denotes differentiation with respect to $\eta, M=\frac{\sigma\left(B_{0}\right)^{2}}{\rho a}$ is the magnetic parameter, $\operatorname{Pr}=\frac{\mu c_{p}}{k}$ is the Prandtl number, $\mu$ is coefficient of viscosity, $R=$ $\frac{16 \sigma^{*} T_{\infty}^{3}}{3 k^{*} k}$ is the thermal radiation parameter and $R e_{x}=\frac{u_{w} x}{v}$ is the local Reynolds number, $D a=\frac{k^{\prime} a}{v}$ is Darcy number, $F=\frac{c_{b}}{\sqrt{k^{\prime}}} x$ is the local inertia coefficient of porous medium (Forchheimer number).

The important physical quantities of interest are the skin friction coefficient $C_{f}$ and the local Nusselt number $N_{u_{x}}$ and defined as

$$
C_{f}=\frac{\tau_{w}}{\rho u_{w}^{2}}, \quad N_{u_{x}}=\frac{x q_{w}}{k\left(T_{w}-T_{\infty}\right)}
$$

Where the skin friction $\tau_{w}$ and the heat transfer from the sheet $q_{w}$ are given by

$$
\begin{array}{r}
\tau_{w}=\mu\left(\frac{\partial u}{\partial y}\right)_{y=0}, \\
q_{w}=-k\left(\frac{\partial T}{\partial y}\right)_{y=0}-\frac{4 \sigma^{*}}{3 k^{*}}\left(\frac{\partial T^{4}}{\partial y}\right)_{y=0} \\
=-\left[k+\left(\frac{16 \sigma^{*} T_{\infty}^{3}}{3 k^{*}}\right)\right]\left(\frac{\partial T}{\partial y}\right)_{y=0}
\end{array}
$$

And $\mu$ and $\mathrm{k}$ are the dynamic viscosity and thermal conductivity respectively. By using equation (5) and (7), we get

$$
C_{f} \sqrt{R e_{x}}=-f^{\prime \prime}(0), \quad \frac{N u_{x}}{\sqrt{R e_{x}}}=-(1+R) \theta^{\prime}(0)
$$

Where $R e_{x}=\frac{u_{w} x}{v}$ is the local Reynolds number.

It is noted that in the absence of magnetic parameter $\mathrm{M}$, thermal radiation $\mathrm{R}$, heat source and sink parameters, $A 1=0$ and $B 1=0$, the closed-form solution for flow problem in terms of Kummer's function were given by Ishak et al. (2009).

\section{NUMERICAL SOLUTION}

An efficient Keller-Box implicit finite difference method has been employed to examine the flow model for the above coupled ordinary differential equations (12) for different values of magnetic parameter M, radiation parameter R, non-uniform heat source or sink parameter, Prandtl number Pr, Darcy number Da and Forchheimer number F of porous medium. The domain of the similarity variable $\eta$ discretized into 0.001 value. The step size and the convergence criteria were taken as $\Delta \eta=0.001$ and $10^{-8}$ respectively. The CPU time to run the matlab code takes 56.42 seconds. The asymptotic boundary conditions are given by eq.(13) were replaced by using a value of 8 for the similarity variable $\eta_{\max }$ as follows:

$$
\eta_{\max }=8, f^{\prime}(4)=\theta(8)=0
$$

\section{RESULTS AND DISCUSSION}

The transformed continuity, momentum and energy equations (12) subjected to the boundary conditions equation (13) were solved numerically by means of implicit finite difference scheme described in Cebeci and Bradshaw (2012). The method has second order convergency, unconditionally stable. A uniform grid is used which is concentrated toward the wall. The calculation is repeated until some convergence criterion is satisfied. In this study the boundary condition for $\eta$ at $\infty$ are replaced by a sufficiently large value of $\eta$ where the velocity and temperature approaches to zero. In order to see the effects of step length $(\Delta \eta)$ we ran the code for two different step size $\Delta \eta=0.001$ and $\Delta \eta=0.01$ in each step length and it has been found good agrement with previous studies available in open litterateur. After some trial we put the maximum value of $\eta$ at $\infty$ of 8 and the grid size of $\Delta \eta=0.01$ We have obtained velocity and temperature profile for different values of governing parameter. The Keller-box method is programmed in MATLAB with the step size of $\Delta \eta=0.01$ in $\eta$. The results obtained are shown through, Figs. 2-5, Figs. 6-11, Figs.12-14 and Figs.15-17 for velocity, temperature, skin friction coefficient and local Nusselt number respectively.

Figs. 2-5 show the velocity graphs for different values of s, M, Da and $\mathrm{F}$ respectively when the other parameters are fixed. Fig. 2 shows the influence of wall transpiration(suction) on the flow velocity. With an increase in suction $(s>0)$ the velocity clearly decrease i.e the flow decelerate. Increasing in suction causes the boundary layer to adhere closer to the flow and reduces momentum transfer.

Fig. 3 reveals the influence of magnetic parameter $M$ on velocity field. It is observed that fluid velocity decreases with an increase in the magnetic parameter $M$. This is because greater retarding effect is generated in the flow with greater values of $\mathrm{M}$ which causes the prominent reduction in velocities due to Lorentz force effect. As magnetic field strength increases the Lorentz force increases which opposes the flow of fluid and flow opposition increase leads to the reduction of velocity of the flow.

Fig.4 depicts the velocity graph for different values in Darcy number Da. In the momentum equation, the Darcian drag term $\frac{1}{D a} f^{\prime}$, is inversely proportional to $\mathrm{Da}$. An increase in Da increases the porous medium permeability and simultaneously decreases the Darcian impedance since progressively less solid fibre are present in the regime. Therefore, the flow 


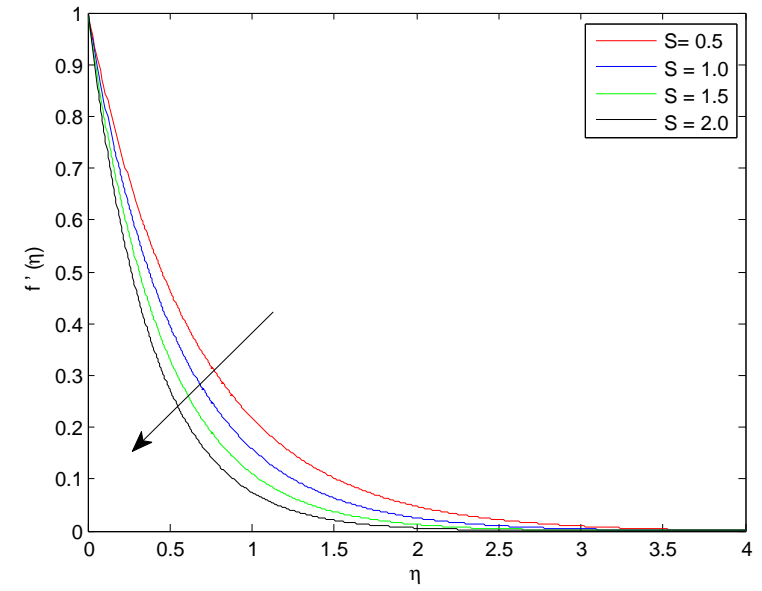

Fig. 2 Velocity profile graph for different values of $\mathrm{S}$, when $M=0.4$, $R=0.3, \operatorname{Pr}=1, A 1=B 1=-0.05, D a=10, F=0.2$

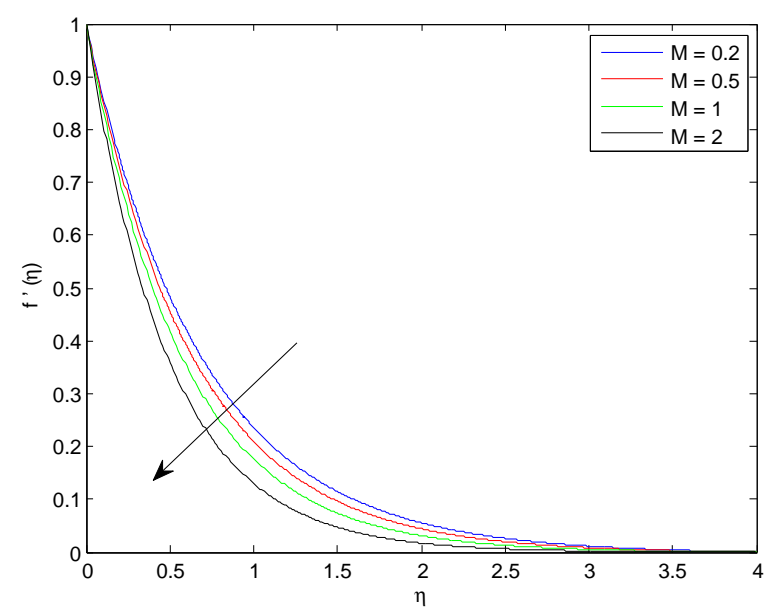

Fig. 3 Velocity profile graph for different values of $\mathbf{M}$, when $s=0.4$, $R=0.3, \operatorname{Pr}=1, A 1=B 1=-0.05, D a=10, F=0.2$

is accelerated for higher Da values causing an increase in the velocity profile. Consequently, the velocity boundary layer thickens increases.

Fig.5 reveals the variation of velocity to a change in Forchheimer inertia drag force parameter. It is observed that the velocity decreases with an increase in the value of Forchheimer number $F$ due to the fact that the second-order quadratic drag force term will increases as a result the impedance offered by fibre of the porous medium will increase which results in decreasing velocity the boundary layer. To summarizes what we discussed on velocity field, the velocity boundary layer thickness decreases monotonically when magnetic parameter M, injection/suction $s$ and Forchheimer number $\mathrm{F}$ increases. Furthermore, the graphs show that velocity gradient on the surface of a stretching sheet decreases with an increase in the values of parameters $\mathrm{M}, \mathrm{F}$ and $\mathrm{S}$.

Fig.6 point up the temperature response to a change in Forchheimer number. The graph shows that as Forchheimer number increase the temperature also increases because as Forchheimer number increase the velocity decreases as wit has been discussed in fig.5. With decelerated flow, heat will be diffused more easily as a result the boundary layer regime will be heat and the boundary layer thickness increased consequently the temperature is increased in the boundary layer.

Fig.7 illustrates the variation of temperature graph for different values of Darcy number Da. From the figure it is possible to see that the temperature decreases with an increase in Da number. This is due to the fact that the presence of fewer solid fibres in the regime with increasing

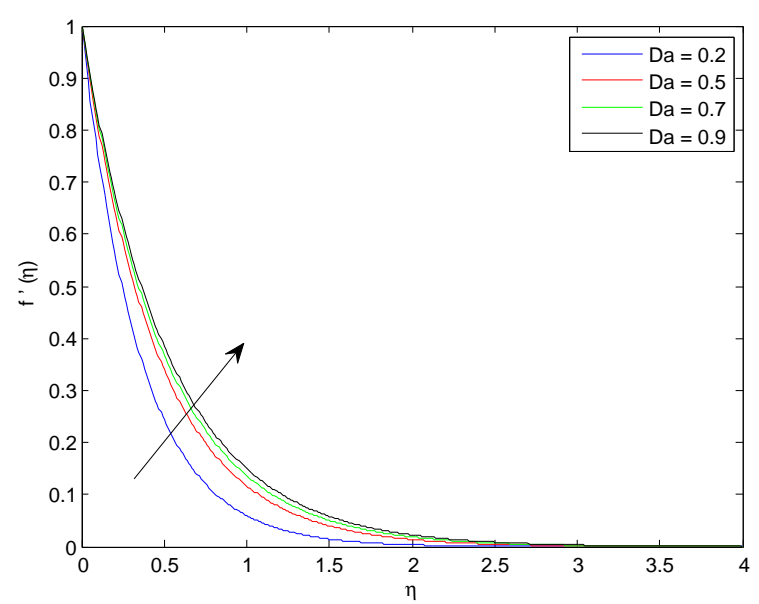

Fig. 4 Velocity profile graph for different values of $\mathrm{Da}$, when $s=0.4$, $R=0.3, \operatorname{Pr}=1, A 1=B 1=-0.05, M=0.5, F=0.2$

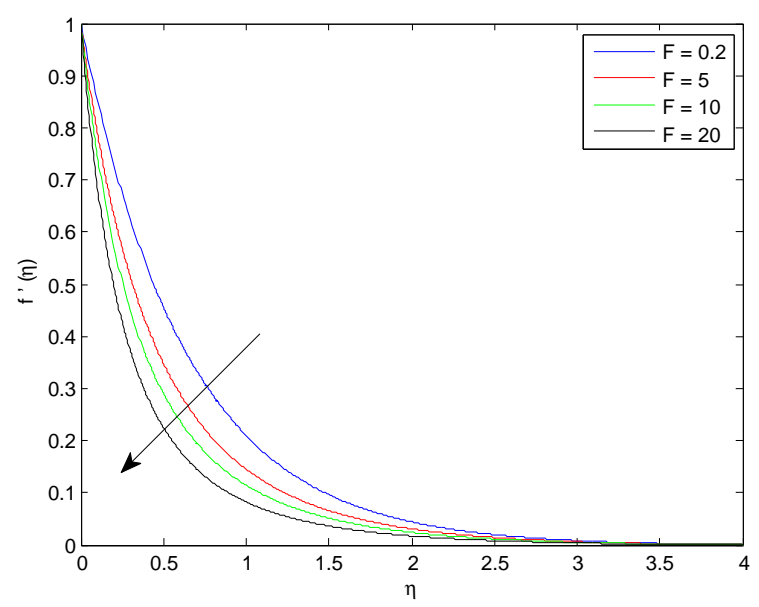

Fig. 5 Velocity profile graph for different values of F, when $s=0.4$, $R=0.3, \operatorname{Pr}=1, A 1=B 1=-0.05, M=0.5, D a=10$

$\mathrm{Da}$, inhibits the thermal conduction in the medium which reduces distribution of thermal energy and thereby decrease thermal boundary layer thickness. This results in the reduction of temperature profile.

Fig. 8 demonstrate the variation of temperature with respect to radiation parameter $R$. It shows that the temperature in the boundary layer increases when the values of radiation parameter $R$ increases at a fixed value of $\eta$. This may be due to the fact that, the divergency of the radiative heat flux $\frac{\partial q_{r}}{\partial y}$ increases as the Rosseland radiative absorptivity $k^{*}$ decreases which in turn increases the rate of radiative heat transfer to the fluid which causes the fluid temperature to increase. This indicate that the effect of thermal radiation is to enhance heat transfer.

Fig.9 depicts the the effects of $\mathrm{B} 1$ on the temperature graph. It is observed that the temperature decreases with an increase in heat $\operatorname{sink}(A 1<$ $0, B 1<0)$ parameter. This is due to the fact that the energy is released when $B 1>0$ which cause the temperature to decrease in the thermal boundary layer. Thus thermal boundary layer thickness decreases with an increase in the heat absorption $\operatorname{sink}(B 1<0)$ parameter.

Fig. 10 shows the variation of temperature profile with respect to coefficient of space-dependent internal heat source or sink parameter $A 1$. The graph reveals that the thermal boundary-layer thickness increases with an increase in the value of $A 1$ at a fixed value for a given $\mathrm{B} 1$ because the boundary layer generate the energy, which causes the temperature to increase with increasing the values of $A 1>0$.

Fig.11 represents the variation of temperature profile with respect 


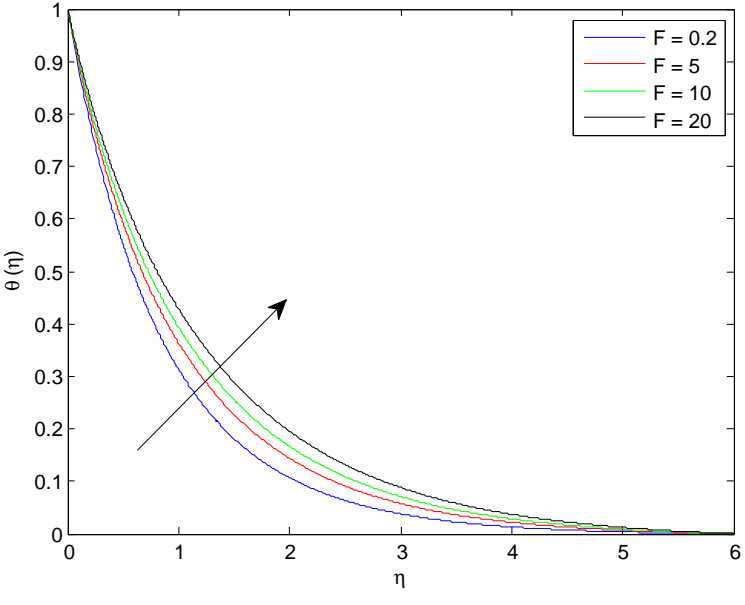

Fig. 6 Temperature profile graph for different values of $\mathrm{F}$, when $s=0.4$, $R=0.3, \operatorname{Pr}=1, A 1=B 1=-0.05, M=0.5, D a=10$

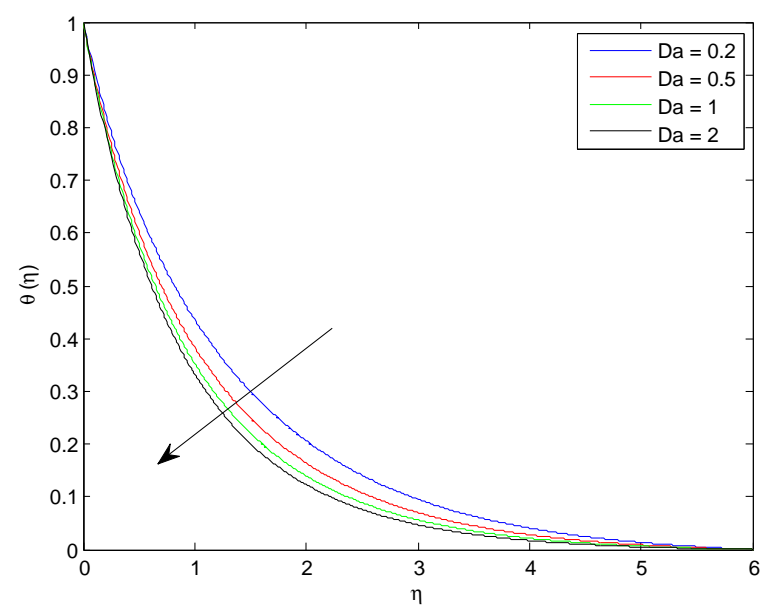

Fig. 7 Temperature profile graph for different values of $\mathrm{Da}$, when $s=0.4$, $R=0.3, \operatorname{Pr}=1, A 1=B 1=-0.5, M=0.5, F=0.2$

to Prandtl number Pr. The graph shows that the temperature graph decreases when the values of Prandtl number Pr increase at a fixed value of $\eta$. This is due to the fact that a higher Prandtl number fluid has relatively low thermal conductivity, which reduces conduction and thereby the thermal boundary layer thickness and as a result temperature decrease.

Figs.12-14 illustrate the effect of $\mathrm{Da}, \mathrm{S}$ and $\mathrm{F}$ on the skin friction coefficient along magnetic field. From all these graphs we can see that the skin friction coefficient increases as the values of magnetic parameter increase. Fig. 12 demonstrates the effect of Darcy number on the skin friction coefficient. The profile reveals that as the value of Darcy number increase, the skin friction decreases. However, the graphs of skin friction coefficient increase as the values of both $\mathrm{F}$ and $\mathrm{s}$ increase. This is illustrated in the figs. 13-14.

Figs.15-17 show the variation of the local Nusselt number along radiation parameter R. The figures show that the local Nusselt number decrease as the influences of radiation parameter increase. Fig. 15 give you an idea about the variation of the local Nusselt number with respect to radiation parameter for varies values of the hear source/sink parameter A1 and B1 when all other governing parameters fixed constant. As the values of $A 1$ increases, the local Nusselt number decrease. This is due to the fact that the space dependent heat source/sink reduces the heat transfer rate. Similarly, fig. 16 illustrates the impacts of time dependent heat source/sink on the local heat transfer rate. Analogous to fig.15, the heat transfer rate decreases as the values of $\mathrm{B} 1$ increase.

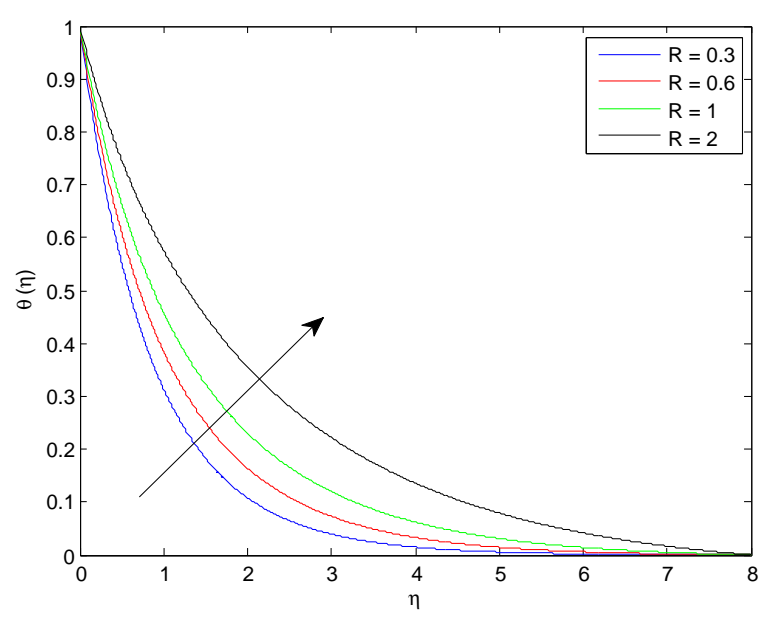

Fig. 8 Temperature profile graph for different values of R, when $s=0.4$, $D a=10, \operatorname{Pr}=1, A 1=B 1=-0.05, M=0.5, F=0.2$

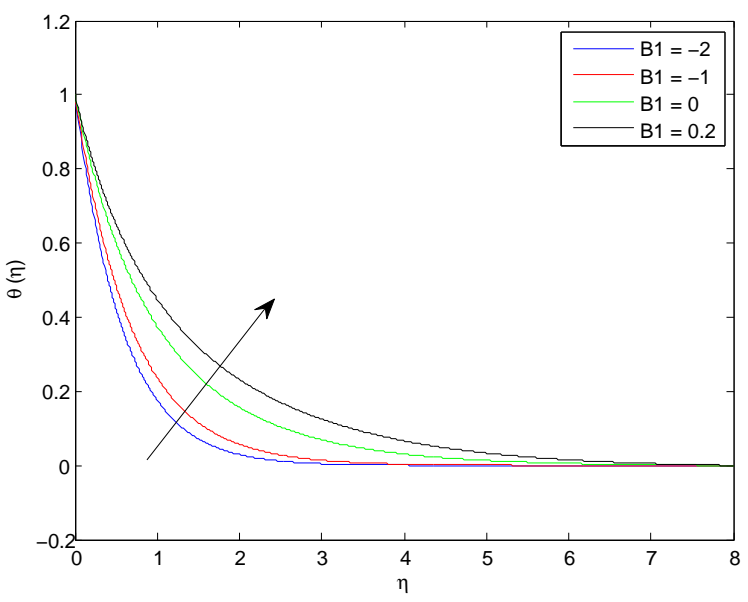

Fig. 9 Temperature profile graph for different values of B1, when $s=0.4$, $D a=10, \operatorname{Pr}=1, A 1=B 1=-0.5, M=R=0.5, F=0.2$

Furthermore, fig. 17 shows the variation of local Nusselt number with respect to Forchheimer number. The graph indicates that the Forchheimer number reduces the local Nusselt number.

To test the accuracy of our method, we have made a comparison of heat transfer at the surface for different values of Pr and s with the result available in the literature as shown in table 1 and the present result is in excellent agreement with previous work.

Table 1 shows a comparison of heat transfer at the surface for different values of $s$ and Pr.The results available in the open literature and ours are found to be in excellent agreement. Hence we can say that the method we used for the present study is appropriate. Besides, the table shows that as both values of $s$ and Pr increase, the local Nusselt number increases.

\section{CONCLUSION}

We examined the problem of MHD boundary layer flow and heat transfer of incompressible, viscous and electrically conducting fluid past a stretching sheet. The influences of thermal radiation and non-uniform heat source/sink is taken into consideration. A numerical method called Keller-box has been employed to study heat transfer in steady MHD boundary-layer flow of continuously stretching permeable surface in the presence of thermal radiation, non-uniform heat source/sink and magnetic field. The effects of various governing physical parameters, such as the magnetic parameter $\mathrm{M}$, radiation parameter $R$, heat source or sink 


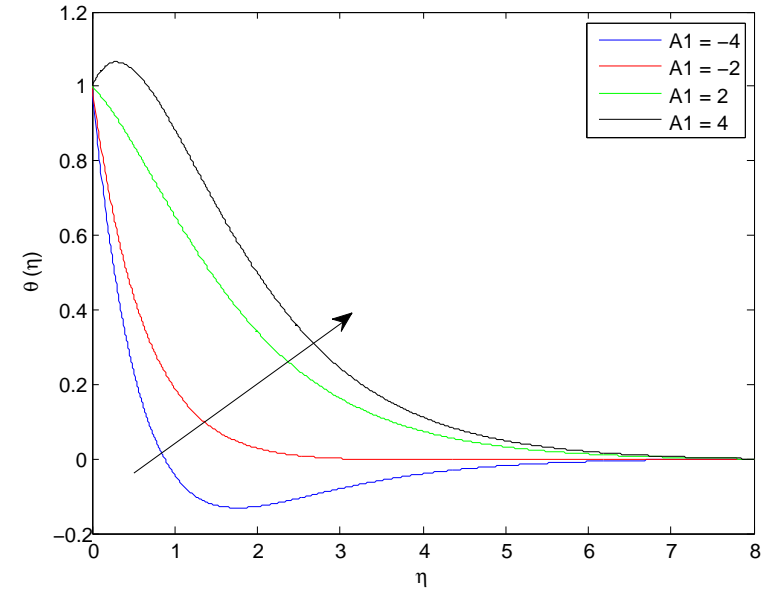

Fig. 10 Temperature profile graph for different values of A1, when $S=$ $0.4, D a=10, \operatorname{Pr}=1, B 1=-0.05, R=0.5, M=0.5$, $F=0.2$

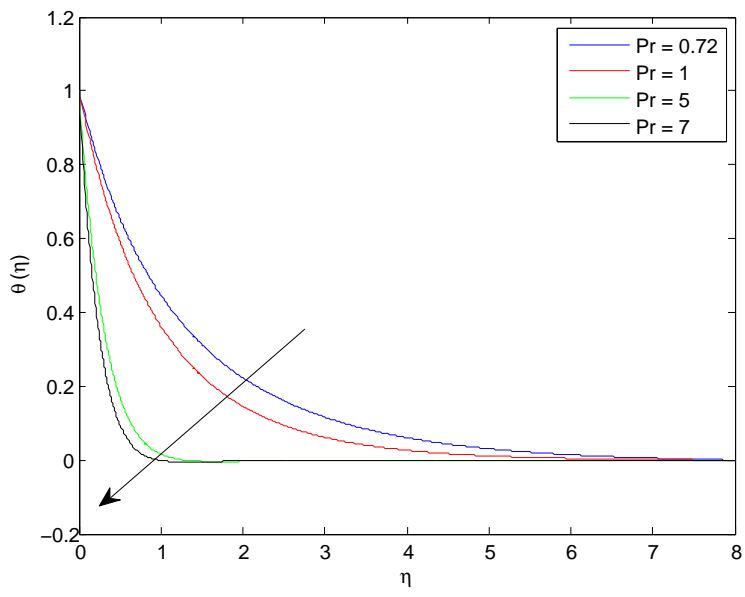

Fig. 11 Temperature profile graph for different values of $\operatorname{Pr}$, when $S=$ $0.4, D a=10, A 1=-0.5, B 1=-0.05, R=0.5, M=0.5$, $F=0.2$

parameter $q_{n}$, Prandtl number Pr, Darcy and Forchhiemer number on the heat transfer characteristic were examined. The numerical results of this study can be summarized as follows:

1. Increasing magnetic field parameter $\mathrm{M}$ and Forchheimer inertial drag parameter $\mathrm{F}$ reduces velocity but increases temperature.

2. Increasing the radiation parameter $\mathrm{R}$ increases temperature.

3. An increment in Darcy number Da increases velocity but reduce temperature.

4. Increasing suction/injection parameters decreases the velocity.

5. The thickness of velocity boundary layer decreases with an increase in suction/injection with internal heat absorbtion and magnetic parameter.

6. Thermal boundary layer thickness decreases with an increase in the value of the magnetic parameter $\mathrm{M}$, suction/injectionparameter $\mathrm{s}$ with internal heat absorbtion parameter, Prandtl number Pr and radiation parameter.

7. The local Nusselt number increases with an increase in the value of radiation parameter $\mathrm{R}$ and magnetic parameter $\mathrm{M}$ for fixed value of Pr.

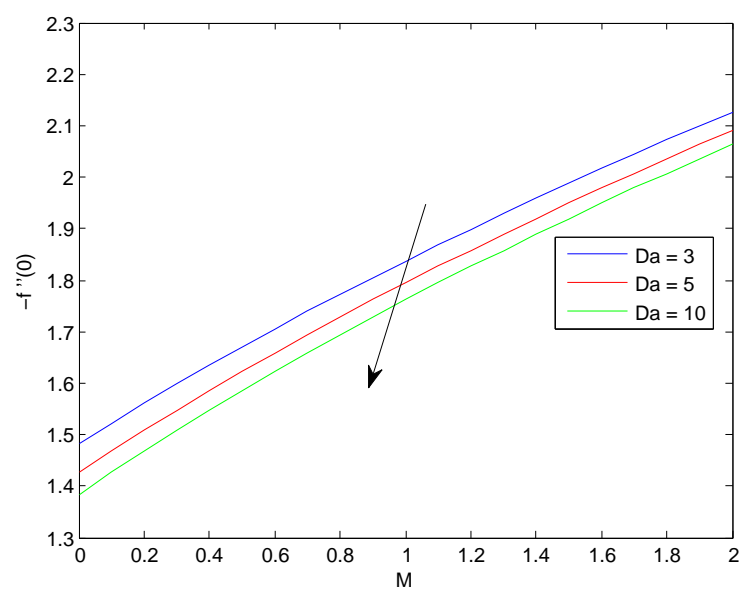

Fig. 12 Skin friction profile graph for different values of Da, along $\mathrm{M}$ when $s=0.4, A 1=-0.5, B 1=-0.05, R=0.5, F=0.2$

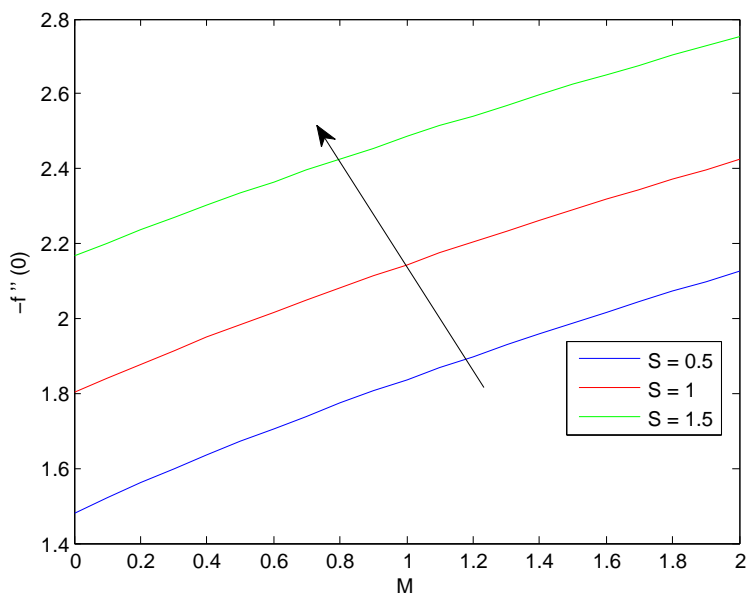

Fig. 13 Skin friction profile graph for different values of $\mathrm{S}$, along $\mathrm{M}$ when $D a=3, A 1=-0.5, B 1=-0.05, R=0.5, F=0.2$

\section{NOMENCLATURE}

$\mathrm{A} 1, \mathrm{~B} 1$

Bo

$\mathrm{M}$

$\mathrm{R}$

$\mathrm{F}$

$\operatorname{Pr}$

$\mathrm{Re}$

$\mathrm{Da}$

$\mathrm{T}$

$N u_{x}$

$c_{p}$

$c_{f}$

$q_{n}$

$q_{r}$

$k^{*}$

$T_{w}$

$T_{\infty}$

$u_{w}$

$v_{w}$

$c_{b}$

$a, b$

$\mathrm{u}, \mathrm{v}$

$\mathrm{k}$
Coefficients of space and temperature-dependent heat source/sink Magnetic field $\left(w b m^{-2}\right)$

Magnetic parameter(Hartman number)

Radiation parameter $\left(W m^{-2} k^{-4}\right)$

Forchheimer number

Prandtl number

Local Reynolds number

Darcy number

Temperature of the fluid inside the boundary layer(K)

Local Nusselt number

Fluid specific heat capacity $\left(J k g^{-1} K^{-1}\right)$

Local skin friction coefficient

Non-uniform heat source/sink

Radiative heat flux

Mean absorption coefficient

Surface temperature of the sheet(K)

Ambient temperature $\left(T_{\infty}<T_{w}\right)(\mathrm{K})$

Stretching velocity of the sheet $\left(m s^{-1}\right)$

Normal velocity at the $\operatorname{surface}\left(m s^{-1}\right)$

Drag coefficient

Empirical constants

Velocity component along $\mathrm{x}$ - and $\mathrm{y}$-direction $\left(m s^{-1}\right)$

Thermal conductivity 


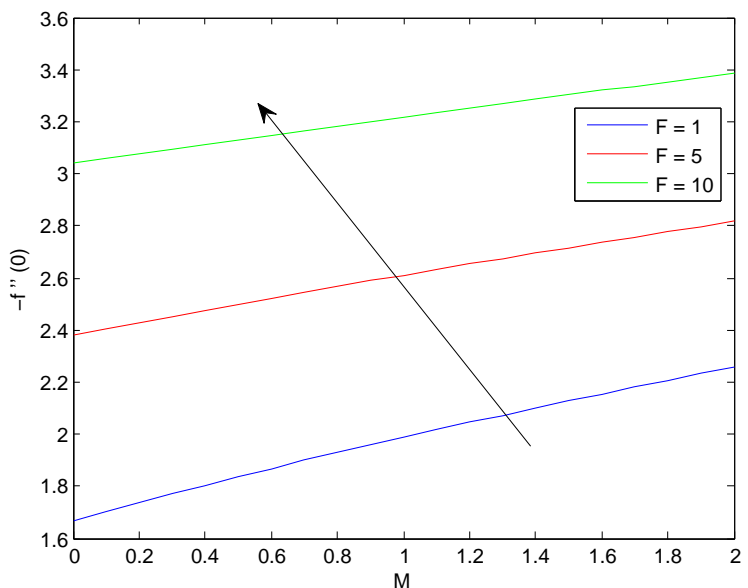

Fig. 14 Skin friction profile graph for different values of $\mathrm{F}$, along $\mathrm{M}$ when $D a=3, A 1=-0.5, B 1=-0.05, R=0.5, s=0.5$

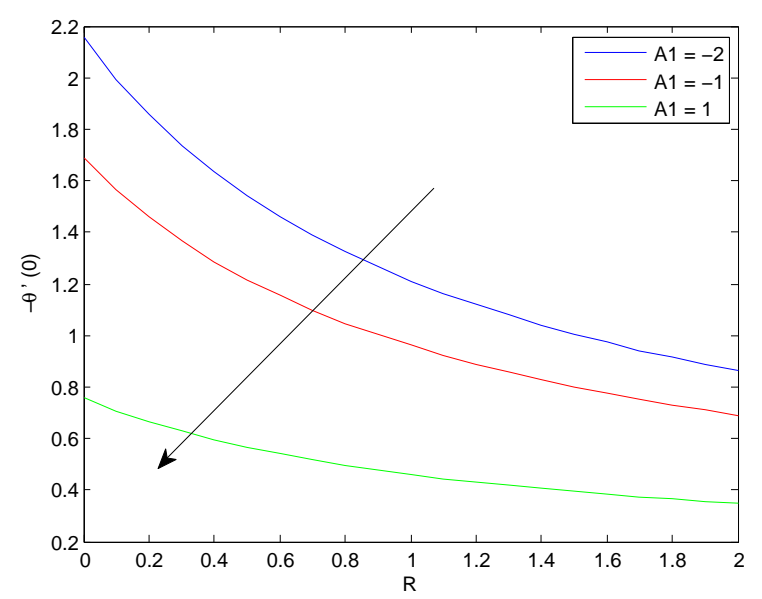

Fig. 15 The graph of local Nusselt number when $D a=3, F=1$, $B 1=-0.05, s=0.5, M=0.5$

$\begin{array}{ll}k^{\prime} & \text { Permeability of porous medium } \\ \mathrm{f} & \text { Dimensionless stream function } \\ \mathrm{s} & \text { Injection/suction parameter } \\ \text { Greeks } & \\ \eta & \text { Dimensionless similarity variable } \\ \mu & \text { Dynamic viscosity of the fluid }(\mathrm{Pa}) \\ v & \text { Kinematic viscosity of the fluid }\left(\mathrm{m}^{2} \mathrm{~s}^{-1}\right) \\ \rho & \text { Density of the fluid }\left(\mathrm{kgm}^{-3}\right) \\ \psi & \text { Dimensional stream function } \\ \theta & \text { Dimensionless temperature } \\ \sigma^{*} & \text { Stephan-Boltzman constant } \\ \sigma & \text { Electrical conductivity } \\ \tau_{w} & \text { Wall shear stress(Pa) } \\ \text { subscripts } & \\ \infty & \text { Condition at the free stream } \\ \mathrm{w} & \text { Condition at the surface }\end{array}$

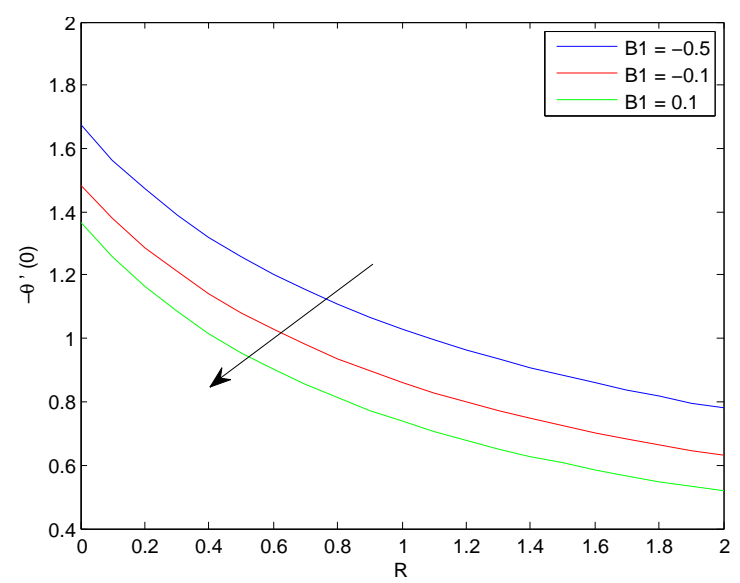

Fig. 16 The graph of local Nusselt number when $D a=3, F=1$, $A 1=-0.5, s=0.5, M=0.5$

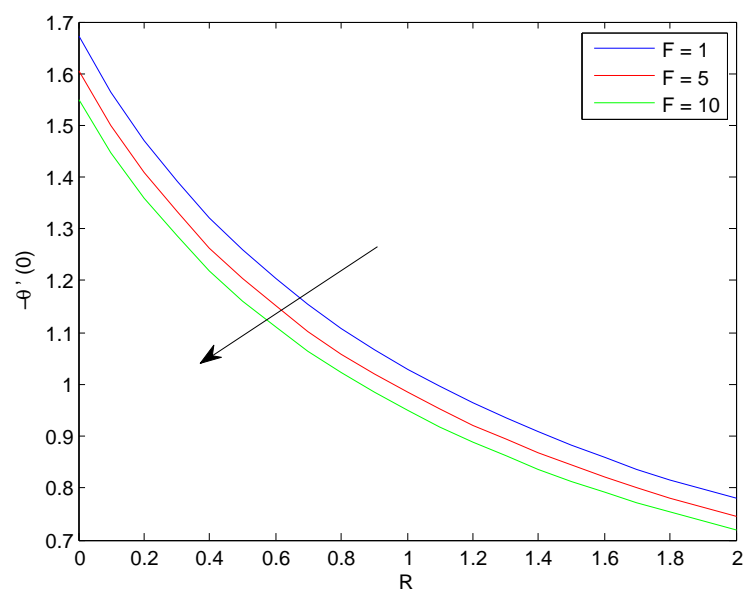

Fig. 17 The graph of local Nusselt number for different values of $F$, along $\mathrm{R}$ when $D a=3, B 1=-0.5, A 1=-0.5, s=0.5, M=0.5$

Table 1 Comparison of values of $-\theta^{\prime}(0)$ for various A,Pr, $M=R=$ $A 1=B 1=F=0.0, D a \rightarrow \infty$ with perviously published data.

\begin{tabular}{lccc}
\hline & \multicolumn{3}{c}{$-\theta^{\prime}(0)$} \\
\hline $\operatorname{Pr}$ & $\mathrm{s}$ & Ishak et al. $(2009)$ & Present result \\
\hline 0.72 & -1.5 & 0.4561 & 0.4561 \\
1 & -1.5 & 0.5000 & 0.5001 \\
10 & -1.5 & 0.6452 & 0.6452 \\
0.72 & 0 & 0.8086 & 0.8086 \\
1 & 0 & 1.0000 & 1.0000 \\
10 & 0 & 3.7207 & 3.7207 \\
0.72 & 1.5 & 1.4944 & 1.4944 \\
1 & 1.5 & 2.0000 & 2.0000 \\
10 & 1.5 & 16.0842 & 16.0842 \\
\hline
\end{tabular}




\section{REFERENCES}

Abel, M.S., and Mahesha, N., 2008, "Heat Transfer in MHD Viscoelastic Fluid Flow over a Stretching Sheet with Variable Thermal Conductivity, Non-Uniform Heat Source and Radiation," Applied Mathematical Modelling, 32(10), 1965-1983.

http://dx.doi.org/10.1016/j.apm.2007.06.038.

Abolbashari, M.H., Freidoonimehr, N., Nazari, F., and Rashidi, M.M., 2014, "Entropy analysis for an unsteady MHD flow past a stretching permeable surface in nano-fluid," Powder Technology, 267, 256-267. http://dx.doi.org/10.1016/j.powtec.2014.07.028.

Cebeci, T., and Bradshaw, P., 2012, Physical and Computational Aspects of Convective Heat Transfer, Springer Science \& Business Media.

Elbashbeshy, E.M., and Aldawody, D.A., 2010, "Heat transfer over an unsteady stretching surface with variable heat flux in the presence of a heat source or sink," Computers \& Mathematics with Applications, 60(10), 2806-2811.

http://dx.doi.org/10.1016/j.camwa.2010.09.035.

Elbashbeshy, E., and Bazid, M., 2004, "Heat Transfer over an Unsteady Stretching Surface," Heat and Mass Transfer, 41(1), 1-4. http://dx.doi.org/10.1007/s00231-004-0520-x.

Elbashbeshy, E., Yassmin, D., and Dalia, A., 2010, "Heat Transfer over an Unsteady Porous Stretching Surface Embedded in a Porous Medium with Variable Heat Flux in the Presence of Heat Source or Sink," African Journal of Mathematics and Computer Science Research, 3(5), 68-73. http://www.academicjournals.org/AJMCSR.

Ibrahim, W., 2016, "Magnetohydrodynamic (mhd) stagnation point flow and heat transfer of upper-convected maxwell fluid past a stretching sheet in the presence of nanoparticles with convective heating," Frontiers in Heat and Mass Transfer (FHMT), 7(1).

http://dx.doi.org/10.5098/hmt.7.4.

Ishak, A., Nazar, R., and Pop, I., 2009, "Heat Transfer over an Unsteady Stretching Permeable Surface with Prescribed Wall Temperature," Nonlinear Analysis: Real World Applications, 10(5), 2909-2913. http://dx.doi.org/10.1016/j.nonrwa.2008.09.010.

Mahmoud, M.A., 2009, "Thermal Radiation Effect on Unsteady MHD Free Convection Flow past a Vertical Plate with Temperature-Dependent Viscosity," The Canadian journal of chemical engineering, 87(1), 47-52. http://dx.doi.org/10.1002/cjce.20135.

Nield, B., 2006, Convection in Porous Media, Springer-Verlag, New York.

Pal, D., 2010, "Magnetohydrodynamic Non-Darcy Mixed Convection Heat Transfer from a Vertical Heated Plate Embedded in a Porous Medium with Variable Porosity," Communications in Nonlinear Science and Numerical Simulation, 15(12), 3974-3987.

http://dx.doi.org/10.1016/j.cnsns.2010.02.003.

Pal, D., 2011, "Combined Effects of Non-Uniform Heat Source/Sink and Thermal Radiation on Heat Transfer over an Unsteady Stretching Permeable Surface," Communications in Nonlinear Science and Numerical
Simulation, 16(4), 1890-1904.

http://dx.doi.org/10.1016/j.cnsns.2010.08.023.

Pal, D., and Chatterjee, S., 2010, "Heat and Mass Transfer in MHD NonDarcian Flow of a Micropolar Fluid over a Stretching Sheet Embedded in a Porous Media with Non-Uniform Heat Source and Thermal Radiation," Communications in Nonlinear Science and Numerical Simulation, 15(7), $1843-1857$.

http://dx.doi.org/10.1016/j.cnsns.2009.07.024.

Pal, D., and Mondal, H., 2010, "Effect of Variable Viscosity on MHD Non-Darcy Mixed Convective Heat Transfer over a Stretching Sheet Embedded in a Porous Medium with Non-Uniform Heat Source/Sink," Communications in Nonlinear Science and Numerical Simulation, 15(6), $1553-1564$

http://dx.doi.org/10.1016/j.cnsns.2009.07.002.

Pop, I., Ishak, A., and Aman, F., 2011, "Radiation effects on the MHD flow near the stagnation point of a stretching sheet: revisited," Zeitschrift für angewandte Mathematik und Physik, 62(5), 953-956.

10.1007/s00033-011-0131-6.

Prasad, K., Pal, D., Umesh, V., and Rao, N.P., 2010, “The Effect of Variable Viscosity on MHD Viscoelastic Fluid Flow and Heat Transfer over a Stretching Sheet," Communications in Nonlinear Science and Numerical Simulation, 15(2), 331-344.

http://dx.doi.org/10.1016/j.cnsns.2009.04.003.

Rashidi, M., Ali, M., Freidoonimehr, N., Rostami, B., and Hossain, M.A., 2014, "Mixed convective heat transfer for MHD viscoelastic fluid flow over a porous wedge with thermal radiation," Advances in Mechanical Engineering, 6, 735939.

http://doi:10.1155/2014/735939.

Rashidi, M., and Erfani, E., 2012, "Analytical method for solving steady MHD convective and slip flow due to a rotating disk with viscous dissipation and Ohmic heating," Engineering Computations, 29(6), 562-579. http://dx.doi.org/10.1016/j.powtec.2014.07.028.

Rashidi, M.M., Momoniat, E., and Rostami, B., 2012, “Analytic approximate solutions for MHD boundary-layer viscoelastic fluid flow over continuously moving stretching surface by homotopy analysis method with two auxiliary parameters," Journal of Applied Mathematics, 2012. http://dx.doi.org/10.1155/2012/780415.

Shateyi, S., and Motsa, S.S., 2010, "Thermal Radiation Effects on Heat and Mass Transfer over an Unsteady Stretching Surface," Mathematical Problems in Engineering. http://dx.doi.org/10.1155/2009/965603.

Siddheshwar, P., and Mahabaleswar, U., 2005, "Effects of Radiation and Heat Source on MHD Flow of a Viscoelastic Liquid and Heat Transfer over a Stretching Sheet," International Journal of Non-Linear Mechanics. http://dx.doi.org/10.1016/j.ijnonlinmec.2004.04.006.

Takhar, H., Bég, O.A., and Kumari, M., 1998, "Computational Analysis of Coupled Radiation-Convection Dissipative Non-Gray Gas Flow in a Non-Darcy Porous Medium Using the Keller-Box Implicit Difference Scheme," International journal of energy research, 22(2), 141-159. http://dx.doi.org/10.1016/j.powtec.2014.07.028. 